\title{
Ein implantierbares Telemetriesystem zur Impedanzspektroskopie
}

\author{
Seeger A. ${ }^{1}$, Kertzscher U. ${ }^{1}$, Gerhäußer A. ${ }^{2}$, Krause F. ${ }^{3}$, Sommer G. ${ }^{3}$, Kolupa J. ${ }^{4}$, Schäfer H. ${ }^{4}$, \\ Arnold R. ${ }^{5}$, Affeld K. ${ }^{1}$, Schaldach M. $^{2}$, Scheel W. ${ }^{3}$, Orglmeister R. ${ }^{4}$, Manck O. ${ }^{5}$ \\ ${ }^{1}$ Humboldt-Universität Berlin, Labor für Biofluidmechanik, Charité, Spandauer Damm 130, D-14050 Berlin \\ ${ }^{2}$ BIOTRONIK GmbH \& Co., Woermannkehre 1, D-12359 Berlin \\ ${ }^{3}$ Fraunhofer Institut für Zuverlässigkeit und Mikrointegration, Gustav-Meier-Allee 25, Gebäude 17, D-13355 Berlin \\ ${ }^{4}$ TU-Berlin, Institut für Elektronik und Lichttechnik, Einsteinufer 17, D-10587 Berlin \\ ${ }^{5}$ TU-Berlin, Institut für Mikroelektronik und Festkörperphysik, Jebensstr. 1, D-10623 Berlin
}

\begin{abstract}
EINLEITUNG
Die kontinuierliche Überwachung des intrakorporalen Zustandes von Geweben beispielsweise zur Erkennung ischämischer Vorgänge nach gefäßchirurgischen Eingriffen oder im Rahmen der Rejektionsdiagnostik läßt sich durch bisher vorhandene Meßsysteme nur bedingt erreichen. Speziell die direkte Erfassung sensitiver Gewebeparameter über einen längeren Zeitraum ohne Belastung für den Patienten stellt in diesem Zusammenhang ein Problem dar.

In der nachfolgenden Arbeit wird das Konzept eines implantierbaren Telemetriesystems vorgestellt, das die Bewertung des Gewebezustandes über die Messung der frequenzabhängigen Bioimpedanz ermöglicht. Besondere Beachtung wird der Auslegung und Umsetzung der einzelnen Systemkomponenten sowie der Vorstellung erster in vitro Messungen zur Evaluierung des Meßsystems geschenkt.
\end{abstract}

\section{METHODE}

Als meßtechnisch effaßbare Größe zur Beschreibung des Gewebezustandes wird die frequenzabhängige elektrische Impedanz herangezogen. Diese spiegelt in ihrem charakteristischen Verlauf die strukturellen und dielektrischen Eigenschaften des Gewebes wider[1]. In einem einfachen Modell läßt sich die Impedanz von Gewebe durch das in Abbildung 1 dargestellte elektrotechnische Ersatzschaltbild annähern. Darin repräsentiert der ohmsche Widerstand $\mathrm{R}_{\mathrm{ex}}$ die Eigenschaften der Elektrolytflüssigkeit des Extrazellulärraums. Die Serienschaltung aus dem "Constant-Phase-Element" CPE und dem ohmschen Widerstand $\mathrm{R}_{\text {in }}$ berücksichtigt das annähernd kapazitive Verhalten der Zellmembran bzw. die Eigenschaften des intrazellulären Fluids. Ändert sich der strukturelle oder funktionelle Zustand des Gewebes, so spiegeln sich diese Ändenungen ebenfalls in den Werten der einzelnen Schaltungselemente wider, wodurch der frequenzabhängige Verlauf der Impedanz beeinflußt wird. Vor allem die Frequenzbereiche der sog. $\alpha$ - und $\beta$-Dispersion $(0,1 \mathrm{~Hz}-10 \mathrm{kHz}$ bzw. $10 \mathrm{kHz}-100 \mathrm{MHz})$ werden als sensitiv gegenüber solchen Zustandsveränderungen des Gewebes angesehen [2], da in diesen Bereichen die
Eigenschaften der Zellmembran bzw. die Kopplung des intra- und extrazellulären Raumes über die Zellmembran in der Impedanz dominiert. Durch die Ermittlung der intrakorporalen Impedanz ist es somit prinzipiell möglich, Gewebeveränderungen zu detektieren und die Impedanz als Indikator für Zustandsänderungen im zugrunde liegenden System heranzuziehen.

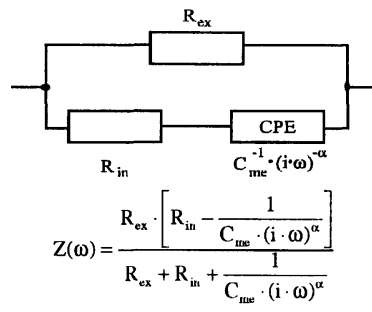

Abbildung 1: Ersatzschaltbild von Gewebe $Z(\omega)$ : frequenzabhängige komplexe Gewebeimpedanz

$R_{e x} \quad:$ Widerstand des extrazellulären Fluids

$R_{\text {in }} \quad:$ Widerstand des intrazellulären Fluids

$C_{m e} \quad:$ Kapazität der Zellmembran

$\alpha \quad$ : Konstante $(0 \leq \alpha \leq 1)$

\section{SYSTEMKONZEPT}

Die Umsetzung dieses Meßverfahrens in ein applizierbares System wird über ein Implantat realisiert, das die Aufnahme der Gewebedaten vornimmt und welches über eine externe Basiseinheit gesteuert wird (Abbildung 2). Beide tauschen Daten über Telemetriemodule aus. Die externe Basiseinheit steht in Verbindung mit einem Zentralcomputer im Krankenhaus. 


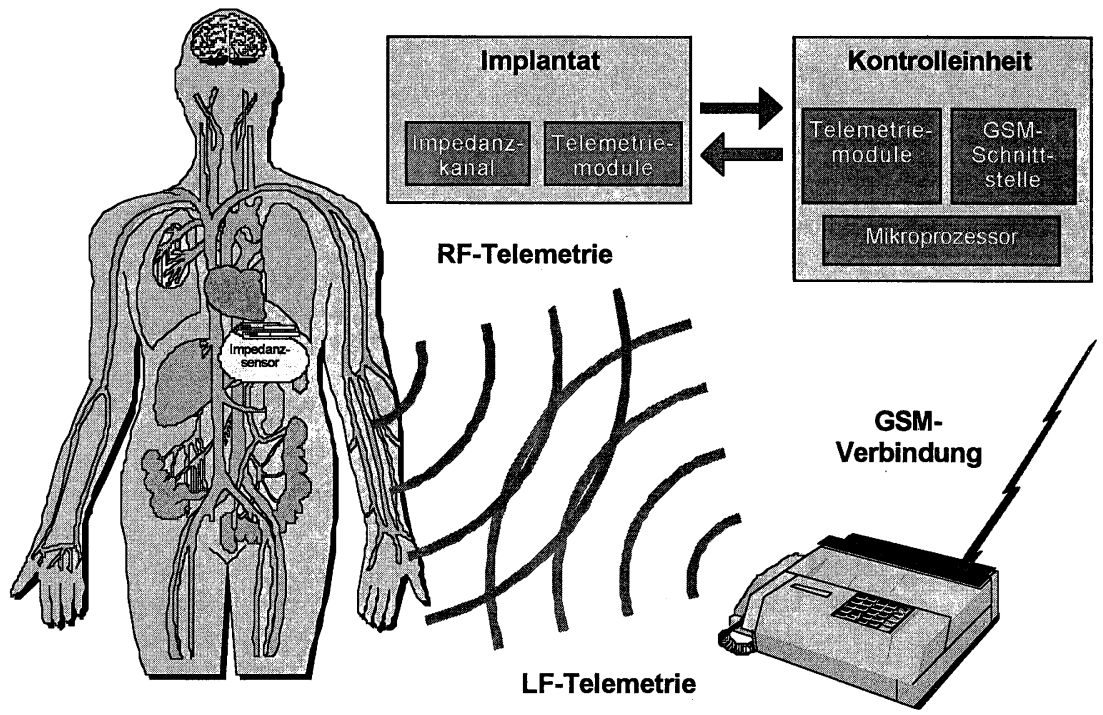

Abbildung 2: Prinzipielles Systemkonzept

\section{IMPLANTAT}

Das Implantat besteht aus folgenden Elementen:

- Energieversorgung

- Meßelektroden

- Meß- und Steuereinheit

- Telemetrieeinheit

Bei der technischen Umsetzung finden erprobte Implantattechnologien Anwendung, um die Rahmenbedingungen hinsichtlich eines möglichst kleinen Volumens und geringem Gewichts des Implantats einzuhalten. Darüberhinaus bieten diese Technologien die Möglichkeit, hybride Materialkonzepte einzusetzen, so daß sich die ebenfalls bestehenden Anforderungen einer hermetischen Kapselung des Implantats bei gleichzeitiger Abschirmung der integrierten Elektronik gegenüber HFStörungen und unter Beachtung der Biokompatibilität der eingesetzten Materialien erreichen lassen.

Die Realisierung des Meßprinzips und der Systemsteuerung im Implantat erfolgt durch die Entwicklung eines applikationsspezifischen integrierten Schaltkreises (ASIC). Dieser nimmt neben der eigentlichen Meßaufgabe ebenfalls die Ansteuerung der Telemetriemodule zum Datenaustausch mit der externen Basiseinheit wahr.

Bei der eigentlichen Messung wird ein Sinusstrom konstanter Amplitude und einer Frequenz zwischen $100 \mathrm{~Hz}$ und $1 \mathrm{MHz}$ in das Gewebe eingeprägt. Dabei wird die am Gewebe abfallende Wechselspannung in Amplitude und Phase gemessen. Zur Bestimmung der einzelnen Impedanzkomponenten aus diesem Meßsignal kommt das in Abbildung 3 dargestellte Verfahren der Synchrondemodulation zum Einsatz. Der Real- und Imaginärteil der Impedanz werden ermittelt, indem die gemessene Spannung mittels eines Signalmischers mit der In-Phase- bzw. Quadratur-Komponente des eingeprägten Wechselstromes in Korrelation gesetzt und anschließend der Gleichanteil über einen Tiefpaß gefiltert wird.

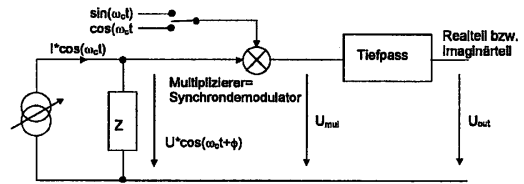

Abbildung 3: Meßprinzip Synchrondemodulation

\section{TELEMETRIEEINHEIT}

Die Telemetrie bildet die Schnittstelle zwischen Implantat und externer Basiseinheit. Die telemetrische Datenübertragung soll bidirektional erfolgen. Die Datenübermittlung vom Implantat zur externen Basiseinheit effolgt im RF-Bereich; die Datenübermittlung zum Implantat im LF-Bereich. 
Folgende Parameter werden angestrebt:

- niedriger Strom- und Volumenbedarf

- raumfüllende Übertragungsreichweite von 2-5 m (davon $0,5 \mathrm{~m}$ durch Körpergewebe)

In der Entwurfsphase geeigneter Schaltungen sowie bei der Entwicklung von Spulengeometrien und zur Frequenzauswahl sind die in Tabelle 1 aufgeführten Einflüsse der Frequenz zu beachten.

Ein wichtiger Punkt bei der Frequenzauswahl ist die Dämpfung im Körpergewebe. Dazu wurden Simulationen durchgeführt, bei den die elektrischen Dämpfungseigenschaften von Muskel- und Körpergewebe analysiert wurden. Die Empfangsleistungen unter den beiden Bedingungen sind in Tabelle 2 aufgeführt. Die realen Empfangsleistungen werden zwischen den Werten von Muskelgewebe und Fettgewebe erwartet.

\begin{tabular}{|l|l|}
\hline Antenne & $\begin{array}{l}\text { Frequenz f hoch: Sendeleistung so- } \\
\text { wie Empfangsleistung steigen }\end{array}$ \\
\hline $\begin{array}{l}\text { Übertragungs- } \\
\text { strecke }\end{array}$ & $\begin{array}{l}\text { Frequenz f hoch: Dämpfung im Kör- } \\
\text { per steigt }\end{array}$ \\
\hline Schaltung & $\begin{array}{l}\text { Frequenz f hoch: Schaltungsaufwand } \\
\text { und Stromverbrauch steigen }\end{array}$ \\
\hline Gesetzgeber & $\begin{array}{l}\text { 402 MHz wird im neuen Frequenz-- } \\
\text { nutzungsplan (1998) für biomedizi- } \\
\text { nische Anwendungen reserviert, nie- } \\
\text { drige Störpegel } \\
\text { LF-Frequenzen (< 150 kHz): offen } \\
\text { zugänglich }\end{array}$ \\
\hline
\end{tabular}

Tabelle 1: Einflußfaktoren auf die Frequenzauswahl

\begin{tabular}{|l|l|l|}
\hline & $\mathrm{f}=100 \mathrm{MHz}$ & $\mathrm{f}=1 \mathrm{GHz}$ \\
\hline Muskelgewebe & $\mathrm{P}=-164 \mathrm{dBm}$ & $\mathrm{P}=-220 \mathrm{dBm}$ \\
\hline Fettgewebe & $\mathrm{P}=-84 \mathrm{dBm}$ & $\mathrm{P}=-63 \mathrm{dBm}$ \\
\hline
\end{tabular}

Tabelle 2: Frequenzabhängige Empfangsleistung unterschiedlicher Gewebearten

Wie bereits oben erwähnt, soll die Datenübertragung von der externen Basiseinheit zum Implantat im LFBereich erfolgen. Bei einer Frequenz von $f=135 \mathrm{kHz}$ schreibt der Gesetzgeber Maximalfeldstärken von $42 \mathrm{~dB} \mu \mathrm{A} / \mathrm{m}$ im Geräteabstand von $10 \mathrm{~m}$ vor. Dies limitiert die maximale Sendeleistung der externen Basiseinheit. Tabelle 3 zeigt die Ergebnisse eines Versuchs, bei dem es zu überprüfen galt, ob für die oben genannte Frequenz eine Reichweite von $5 \mathrm{~m}$ mit ausreichender Störunempfindlichkeit realisiert werden kann. Bei beiden Versuchen wurde die Maximalfeldstärke eingehalten. Der Versuchsaufbau ist in Abbildung 4 dargestellt. Es wurde herausgefunden, daß mit einer Empfangsantenne mit einem Radius von $0,5 \mathrm{~cm}$ und 60 Windungen und unter Berücksichtigung des Umgebungsrauschens und der oben genannten Maximalfeldstärke eine ausreichend große Empfangsspannung von $31 \mathrm{~dB} \mu \mathrm{V}$ detektiert werden kann.

Für die Signalübertragung vom Implantat zur Patienteneinheit ist die vom Gesetzgeber reservierte Frequenz von $402 \mathrm{MHz}$ (RF-Bereich) vorgesehen. Die Daten werden frequenzmoduliert übertragen. Die Antenne wird außerhalb des Titangehăuses plaziert. Messungen an einem
Prototypen ergaben die in Abbildung 5 dargestellten richtungsabhängigen Empfangsspannungen. Als Empfangsantęnne diente zur Referenz ein $\lambda / 2$ Dipol.

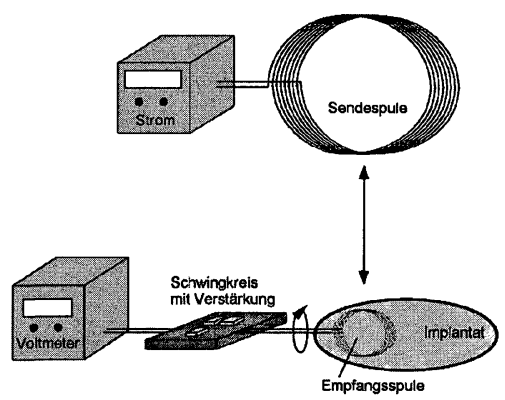

Abbildung 4: Testaufbau für Implantatempfänger

\begin{tabular}{|l|l|l|l|}
\hline $\mathrm{Nr}$. & $\mathrm{I}_{\text {sende }}$ & $\Delta \mathrm{S}$ & $\mathrm{U}_{\text {empf }}$ \\
\hline 1 & $1 \mathrm{~A}$ & $5 \mathrm{~m}$ & $31 \mathrm{~dB} \mu \mathrm{V}$ \\
\hline 2 & $1 \mathrm{~A}$ & $3 \mathrm{~m}$ & $39 \mathrm{~dB} \mu \mathrm{V}$ \\
\hline
\end{tabular}

Tabelle 3: Empfangsspannung bei $135 \mathrm{kHz}$ unter Berilcksichtigung der maximal erlaubten Sendeleistung

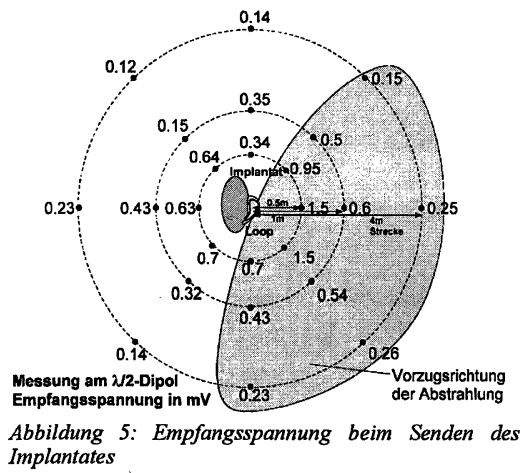

Mit den bisherigen Meßergebnissen zur telemetrischen Datenübertragung wurde das Konzept der Zweifrequenztelemetrie als tragfähig bewertet. Zur Erhöhung der Leistungsfähigkeit der Telemetrie und damit zur zuverlässigen Realisierung der Systemanfordenungen muß noch vorhandenes Optimierungspotential bezuiglich der dämpfungsabhängigen Richtcharakteristik und des Antennendesigns in weiterführenden Arbeiten umgesetzt werden 


\section{EXTERNE BASISEINHEIT}

Die externe Basiseinheit stellt das Bindeglied zwischen dem Implantat und dem zentralen Überwachungsrechner im Krankenhaus oder einer Arztpraxis dar.

Die Basiseinheit besteht aus folgenden Modulen:

- HF-Empfänger

- LF-Sender

- optional LF-Empfänger

- Mikrocontrollermodul mit Interface-Logik

- externe serielle Schnittstelle zum direkten Anschluß an einen PC/Laptop, eventuell als USB

- interne serielle Schnittstelle zum Anschluß wahlweise eines Analogmodems oder eines GSM-Modules.

- Stromversorgung

- Busschnittstelle zur Vemetzung mehrerer Geräte

Den Aufbau der externen Basisstation zeigt Abbildung 6. Die Kommunikation zwischen der Basiseinheit und dem Überwachungsrechner findet über das Telefonnetz (GSM, analog oder DECT) statt, wenn die Basiseinheit im Hause des Patienten arbeitet. In der Arztpraxis kann zur direkten Kommunikation die serielle Schnittstelle verwendet werden, in Krankenhäusern empfiehlt sich entweder die Verwendung der Haustelefonanlage oder eines LAN, um mehrere Basisstationen verwalten zu können. Als LAN kann z.B. ein CAN-Bus verwendet werden

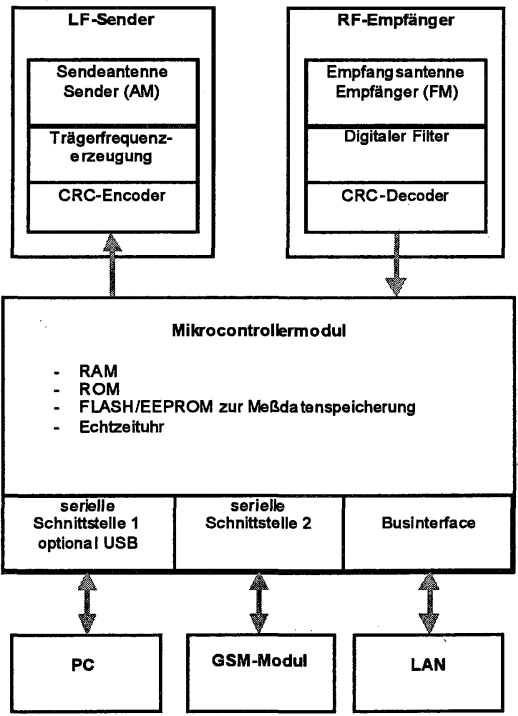

Abbildung 6: Aufbau der externen Basiseinheit
In Abbildung 7 ist der prinzipielle Ablauf einer Impedanzmessung dargestellt. Um eine Messung zu starten, wird zunächst das Implantat von der Patienteneinheit durch das Senden des Wake-Up Befehls aktiviert und eine bidirektionale Telemetrie mit dem Implantat aufgebaut. Dies bedingt allerdings, daß sich der Patient in der Telemetriereichweite der Patienteneinheit befinden muß. Anschließend werden die zur Messung benötigten Parameter telemetrisch an das Implantat übertragen.

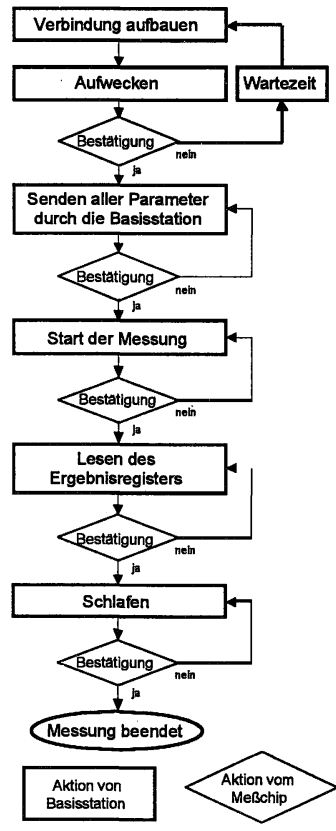

\section{Abbildung 7: Prinzipieller Ablauf einer Messung}

\section{BIOIMPEDANZMESSUNGEN}

Zur Evaluierung des Meßprinzips und zur Spezifikation der Anforderungen an den Sensorchip wurden zunächst Bioimpedanzmessungen in vitro an perfundierten Schweinenieren und einer Schweineleber bzw. in vivo an einer Niere eines anästhesierten Hundes durchgeführt. Hierfür wurde ein Impedanzmeßgerät entwickelt, welches die Phasenverschiebung und den Betrag der Impedanz bei Frequenzen zwischen $10 \mathrm{~Hz}$ und $300 \mathrm{kHz}$ mißt. Die Messungen wurden mit einem 2-Elektroden-System durchgeführt. Für spätere Messungen soll auf ein 4-Elektroden-System übergegangen werden, da bei solch einem System der Übergangswiderstand zwischen Organ und Elektroden vernachlässigt werden kann. 
Der Einfluß der verschiedenen Elektrodenparameter (Größe, Beschichtung und Abstand) auf die Impedanz wurde untersucht. Die Messungen an nicht perfundierten Schweinenieren zeigten, daß der Einfluß der Elektrodengröße auf die Impedanz stärker ist als der Einfluß des Elektrodenabstands. Zwei verschiedene Beschichtungsarten wurden untersucht: fraktal mit Iridium bzw. mit Iridiumoxid. Durch diese Beschichtung läßt sich die Polarisation im Vergleich zu unbeschichteten Elektroden vermindem [3]. Der Einfluß der Beschichtungsart ist relativ klein. Qualitativ ähnliche Ergebnisse lieferten Impedanzmessungen an unter physiologischen Bedingungen hämoperfundierten Organen.

Zwei Ergebnisse von Bioimpedanzmessungen zeigen die Abbildungen 8 und 9. Alle Messungen wurden mit fraktal mit Iridium beschichteten Plattenelektroden mit einem Durchmesser von $7 \mathrm{~mm}$ durchgeführt. Abbildung 8 zeigt die Ergebnisse einer Messung an der Niere eines Hundes in vivo. Die Impedanz und die Phasenverschiebung sind in Abhängigkeit von der Frequenz und zu zwei verschiedenen Zeitpunkten dargestellt (Zeitdifferenz: zwei Stunden). Bei der ersten Messung arbeitete die Niere unter physiologischen Bedingungen, bei der zweiten Messung lag Ischämie vor. Man sieht einen deutlichen Unterschied zwischen den Graphen beider Zustände. Das Ergebnis, daß die Impedanz im ischämischen Zustand höher ist als im gesunden, wird z.B. in [4] und [5] bestätigt.

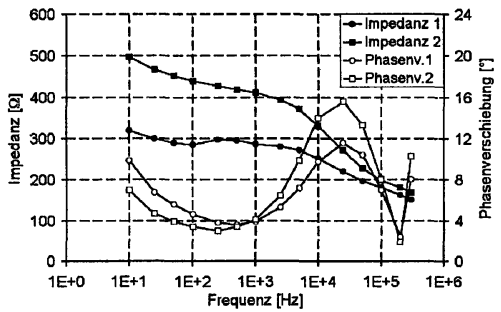

Abbildung 8: Die Bioimpedanz einer Hundeniere in vivo. Dargestellt sind der Betrag der Impedanz und die Phasenverschiebung in Abhängigkeit von der Frequenz und zu zwei verschiedenen Zeitpunkten. Beim Zeitpunkt 1 arbeitete die Niere unter physiologischen Bedingungen; beim Zeitpunkt 2 lag Ischämie vor. Die Zeitdifferenz zwischen den beiden Messungen betrug zwei Stunden.

Abbildung 9 zeigt die Impedanz und die Phasenverschiebung gemessen an einer hämoperfundierten Schweineleber zu zwei verschiedenen Zeitpunkten (Zeitdifferenz: anderthalb Stunden) bei nahezu gleichen Bedingungen

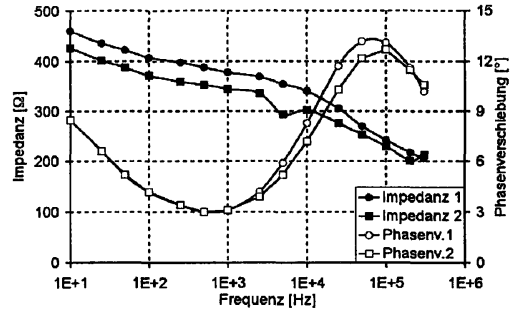

Abbildung 9: Die Bioimpedanz einer hämoperfundierten Schweineleber. Dargestellt sind der Betrag der Impedanz und Phasenverschiebung in Abhängigkeit von der Frequenz und zu zwei verschiedenen Zeitpunkten. Die physiologischen Bedingungen beider Messungen waren nahezu gleich. Die Zeitdifferenz zwischen den beiden Messungen betrug anderthalb Stunden.

\section{DISKUSSION}

Mit dem vorgestellten System soll eine intrakorporale Impedanzmessung zur Gewebezustandsbewertung bei Transplantationspatienten telemetrisch ermöglicht werden. Für das Implantat und die externe Basiseinheit wurden die Anfordenungen festgelegt und ein Realisierungskonzept entwickelt. Die vorgesehene Telemetrieeinheit wurde auf ihre Einsetzbarkeit geprüft und für die speziellen Anforderungen des Anwendungsgebietes weiterentwickelt. Nächster Schritt ist die Zusammenführung der einzelnen Systemkomponenten. Die Bioimpedanzmessungen an Gewebe zeigten eine gute Übereinstimmung mit Daten aus der Literatur. Die Datenbasis ist aber noch zu klein, um eine Korrelation der Messungen mit dem Nierenzustand zu erreichen. Insbesondere wurde noch nicht die Abstoßungsreaktion des Körpers auf das implantierte Organ untersucht

\section{FÖRDERUNG}

Diese Arbeit wird gefördert durch das Land Berlin im Rahmen des Förderprogrammes "Informations- und Kommunikationstechnologie (IKT)".

\section{LITERATUR}

[1] Foster K. R, Schwan H.P.: Dielectric Properties of Tissues and Biological Materials: A Critical Review, Critical Reviews in Biomedical Engineering 17, 1989, 25-104

[2] Rigaud B., Morucci J.P., Chauveau N.: Bioelectrical Impedance Techniques in Medicine Part I: Bioimpedance Measurement - Second Section: Impedance Spectroscopy, Critical Review in Biomedical Engineering 24, 1996, 257-351 
[3] Schmidt K., Fröhlich R., Bolz A., Schaldach M.: Effect of Surface Morphology on Electrode Impedance - A New Approach to a Low Polarisation Electrode, Proceedings of the IX. International Conference on Electrical BioImpedance, Heidelberg, 1995, 36-39

[4] Casas O., Bragós R., Riu P.J., Rosell J., Tresánchez M., Warren M., Rodríguez-Sinovas A., Carreño A., Cinca J.: In-vivo and in-situ characterisation using electrical impedance spectroscopy, Proceedings of the X. International Conference on Electrical Bio-Impedance, 1998, Barcelona, ISBN 84-7653-686-0

[5] Gheorghiu M., Gersing E., Gheorghiu E.: Quantitative analysis of impedance spectra of organs during ischemia, Proceedings of the $\mathrm{X}$. International Conference on Electrical BioImpedance, 1998, Barcelona, ISBN 84-7653-686-0 